\title{
Microbial ecosystem of processing units during production process of Petrovská klobása
}

\author{
Janković Vesna ${ }^{1}$, Petrović Ljiljana ${ }^{2}$, Džinić Natalija $^{2}$, Lakićević Brankica $^{1}$, Šarić Ljubiša ${ }^{3}$, Škaljac Snežana ${ }^{2}$, \\ Šojić Branislav ${ }^{2}$, Jokanović Marija ${ }^{2}$
}

A b s tr a c t: Production of traditional dry-fermented sausages is associated with natural contamination by environmental flora. This microbiota is usually referred to as "house flora". This contamination occurs during slaughtering and increases during manufacturing. The diversity of microbiota in small-scale processing units, during production process of the traditional fermented dry sausages - Petrovská klobása, is reviewed in the present paper. Test samples were collected in two village households in Bački Petrovac, where the preparation of Petrovská klobása samples was performed in a traditional manner. The total number of 43 samples was subjected to laboratory examination. Generally, before stuffing, Listeria monocytogenes and Staphylococcus aureus were detected in 6.97 and $9.30 \%$, respectively. Escherichia coli was found in $18.60 \%$. The tested samples of end product at the end of the storage period $\left(270^{\text {th }}\right.$ day) were safe with presence of bacteria populations from the working environment, such as: aerobic bacteria, Micrococcaceae, Lactic acid bacteria and Enterococcus spp. Examination of the hygienic status of the food processing environment, equipment, raw materials and final product provides an overview of growth trends and the disappearance of bacterial populations.

Key words: Petrovská klobása, house flora, processing environment, growth trends.

\section{Introduction}

In many European countries, the demand for traditional food products has increased. Moreover, food and gastronomy form an inherent link with tourism in Europe, with a renewed interest of consumers in typical and regional food. Petrovská klobása, traditional and autochthonous dry - cured sausage, presents a part of gastronomic heritage of Slovaks in Vojvodina. Nowadays, they are manufacturing the product in a traditional way according to the original recipe of their ancestors, without the use of nitrate/ nitrite, glucono delta-lactone (GDL) and microbial starters. In rural households, in the Municipality of Bački Petrovac, this sausage is made by the end of November and during December. Petrovská klobá$s a$ is made by mixing partly cooled (cca 4 h p.m) or cold (cca 24 h p.m) medium chopped lean pork and fat (up to $10 \mathrm{~mm}$ ) with addition of powdered red hot spicy paprika, salt, crushed garlic, caraway and sugar. A well-mixed filling, which is prepared within
15-30 minutes by using a unique technique of manual mixing with kneading and overturning, is stuffed into natural casings consisting of the rear part of pig intestines (colon), forming units $35-45 \mathrm{~cm}$ long and $4.5-5.0 \mathrm{~cm}$ in diameter. After stuffing, the sausages are left to drain for a while and then subjected to cold smoking for about 10-15 days, using specific kinds of wood (cherry wood in particular). When the smoking process is finished, the sausage is kept in a dry and well ventilated place to dry and ripen, until it achieves an optimum quality, which takes about four months (Tasić, 2012; Janković, et al., 2013; Šojić et al., 2014). Petrovská klobása is a product of protected geographical origin, under number 44, based on the order issued by the Republic Bureau for Intellectual Property, number 9652/06 G-03/06, on $21 / 05 / 2007$. In order to achieve a recognizable product of standardized supreme quality which will be continually produced in the controlled conditions, the aim of this study is to determine the parameters of typical house flora during the production

Acknowledgements: The research was financially supported by the Ministry of Education, Science and Technological Development, Republic of Serbia, project TR31032.

\footnotetext{
${ }^{1}$ Institute of Meat Hygiene and Technology, Kaćanskog 13, 11000 Belgrade, Republic of Serbia;

${ }^{2}$ University of Novi Sad, Faculty of Technology Novi Sad, Bulevar cara Lazara 1, 21000 Novi Sad, Republic of Serbia;

${ }^{3}$ Institute of Food Technology, Bulevar cara Lazara 1, 21000 Novi Sad, Republic of Serbia.
}

Corresponding author: Vesna V. Janković, vessna@inmesbgd.com 
process of Petrovská klobása, which is crucial because of the safety of the final product.

\section{Materials and methods}

\section{Samples}

Test samples were collected from two village households (A and B) in Bački Petrovac, where the preparation of Petrovská klobása samples was performed in a traditional manner. Testing included examination of 43 samples, such as: swabs - workers' hands $(n=11)$, working surfaces $(n=1)$, equipment before beginning the operation $(n=9)$, equipment after the operation $(n=9)$, and other swabs from the working area - wall, drains etc. $(n=7)$ and samples of fillings $(n=2)$ and sausages after the drying process $(n=4)$. The sample area was swabbed using a maximum recovery diluent (MRD, Oxoid) moistened cotton swab. The samples were stored at $0-4^{\circ} \mathrm{C}$ (coolbox) and then transported to the laboratory under chilled conditions $0-4^{\circ} \mathrm{C}$. The samples were examined within 24 hours.

Each sample was tested on the presence of the set of the following bacteria: (1) Total Viable Count/ TVC (SRPS ISO 4833-1; Plate Count Agar -PCA, Oxoid); incubated at $30^{\circ} \mathrm{C}$ for $72 \mathrm{~h}$; (2) Total bacterial count of the Micrococaceae family (Manitol salt phenol - red agar, Oxoid), incubated at $30^{\circ} \mathrm{C}$ for $72 \mathrm{~h}$; (3) Total Enterobacteriaceae count (SRPS ISO 21528-2; Violet Red Bile agar with glucose - VRBG, Oxoid), incubated at $30^{\circ} \mathrm{C}$ for $72 \mathrm{~h}$; (4) Total count of $\beta$ - glucuronidase positive $E$. coli (SRPS ISO 16649-2; Tripton Bile x Glucuronide agar/TBX, Oxoid), incubated at $44^{\circ} \mathrm{C}$ for $24 \mathrm{~h}$; (5) Enterococcus spp. counts (Bile esculin azide agar, Biokar diagnostics), incubated at $37^{\circ} \mathrm{C}$ for $48 \mathrm{~h}$; (6) Total count of coagulase positive staphylococci (SRPS ISO 6888 - 1, Baird Parker, Oxoid), incubated at $37^{\circ} \mathrm{C}$ for $24 \mathrm{~h}$; (7) Pseudomonas spp. counts, (Pseudomonas Selective Agar - Cetrimide Agar, Merck), incubated at $35^{\circ} \mathrm{C}$ for $48 \mathrm{~h}$; (8) Total count of sulphate-reducing bacteria, which grow in anaerobic conditions (SRPS ISO 15213; Iron Sulfite Agar, Oxoid), incubated at $37^{\circ} \mathrm{C}$ for $48 \mathrm{~h}$; (9) Total count of Clostridium perfringens (SRPS ISO 7937; Sulfite cycloserine Agar, Oxoid), incubated at $37^{\circ} \mathrm{C}$ for 20 h; (10) Salmonella spp. (SRPS ISO 6579; modified Rappaport Vasilidis Soft Agar), incubated at $42^{\circ} \mathrm{C}$ for $24 \mathrm{~h}$; (Rambach, Merck), incubated at $37^{\circ} \mathrm{C}$ for $24 \mathrm{~h}$; (11) Lactic acid bacteria presence in samples of chunk meat and filling (ISO 15241; Man-Ragosa Sharpe/MRS, Merck, Darmstad, Germany), incubated at $30^{\circ} \mathrm{C}$ for $48-72$; (12) Listeria monocytogenes presence and total count (SRPS ISO 11290-1, 2; ALOA, Merck).

\section{Immunoenzymatic assay}

For detection of Listeria monocytogenes, a Vidas - L. monocytogenes Xpress (LMX, BioMérieux) was used. In case of food samples, $25 \mathrm{~g}$ of sample (analytical unit) was aseptically added to $225 \mathrm{~mL}$ of LMX broth in a stomacher bag (Seward). In case of environmental samples, for each swab, $10 \mathrm{~mL}$ of LMX broth was aseptically added for each swab. Incubation period was $30 \pm 1^{\circ} \mathrm{C}$ for $22-24 \mathrm{~h}$ for food samples or $24-26 \mathrm{~h}$ for environmental samples. After a specific period of incubation, 1-2 $\mathrm{ml}$ broth was removed into a sterile test-tube (Sigma Aldrich), which was heated at 95 to $100^{\circ} \mathrm{C}$ for $5 \pm 1 \mathrm{~min}$. The tube was cooled down and $250 \mu \mathrm{l}$ of the enriched sample was taken to test. All positive results obtained were confirmed by the reference SRPS ISO 11290-1 method or by using the ALOA chromogenic agar.

\section{Statistical analysis}

Statistical analysis was carried out using STATISTICA 9.1 (StatSoft, Inc., Tulsa, OK, USA). All data were presented as a mean value with the standard deviation indicated (mean $\pm \mathrm{SD}$ ).

\section{Results}

Results of testing are presented in Tables 1, 2, 3, 4 and 5. The environment of processing units was colonized at variable levels by resident spoilage and technological microbiota, with sporadic contamination by pathogenic microbiota. In the households $\mathrm{A}$ and B (Tables 1, 2, 3 and 4), the presence of aerobic bacteria, E. coli, enterococci, Staphylococcus aureus, Enterobacteriaceae and Listeria spp. was detected. In household A (Table 1), the aerobic bacteria counts ranged from $1.26 \pm 0.17 \log _{10} \mathrm{cfu} / \mathrm{cm}^{2}$ (knife) up to $8.04 \pm 0.91 \log _{10} \mathrm{cfu} / \mathrm{cm}^{2}$ (saw after cutting). E. coli was present in two samples (saw after cutting and table), while enterococci were found in all experimental samples, with a range between $2 \pm 0 \log _{10} \mathrm{cfu} / \mathrm{cm}^{2}$ (workers hands) and $5.67 \pm 0.06 \log _{10} \mathrm{cfu} / \mathrm{cm}^{2}$. (workers' hands after slaughtering). Staphylococcus aureus was found in only one sample (workers' hands). Enterobacteriaceae had total counts that ranged between $2.67 \pm 0.31 \log _{10} \mathrm{cfu} / \mathrm{cm}^{2}$ (table) and saw after cutting $\left(5.04 \pm 0.4 \log _{10} \mathrm{cfu} / \mathrm{cm}^{2}\right)$. Other groups of bacteria were not detected. Household 
Table 1. Microbiological contamination of processing environment, food contact surfaces, equipment and workers' hands in the household A during the meat production process $\left(\mathrm{X} \pm \mathrm{SD}, \log _{10} \mathrm{cfu} / \mathrm{cm}^{2}\right)$.

Tabela 1. Mikrobiološka kontaminacija radne sredine, radnih površina, pribora i ruku radnika u okviru domaćinstva A tokom proizvodnje mesa $\left(\mathrm{X} \pm \mathrm{SD}, \log _{10} \mathrm{cfu} / \mathrm{cm}^{2}\right)$

\begin{tabular}{|c|c|c|c|c|c|c|c|c|}
\hline $\begin{array}{l}\text { Microbiological } \\
\text { contamination/ } \\
\text { Mikrobiološka } \\
\text { kontaminacija }\end{array}$ & $\begin{array}{c}\text { Workers' } \\
\text { hands/ } \\
\text { Ruke } \\
\text { radnika }\end{array}$ & $\begin{array}{c}\text { Workers' } \\
\text { hands after } \\
\text { slaughtering/ } \\
\text { Ruke radnika } \\
\text { posle klanja }\end{array}$ & $\begin{array}{c}\text { Saw/ } \\
\text { Testera }\end{array}$ & $\begin{array}{c}\text { Saw after } \\
\text { cutting/ } \\
\text { Testera } \\
\text { posle klanja }\end{array}$ & Knife/Nož & $\begin{array}{l}\text { Knife after } \\
\text { cutting/ } \\
\text { Nož posle } \\
\text { klanja }\end{array}$ & $\begin{array}{c}\text { Table/Radna } \\
\text { površina }\end{array}$ & $\begin{array}{c}\text { Wall/ } \\
\text { Zid }\end{array}$ \\
\hline $\begin{array}{l}\text { Total viable count/ } \\
\text { Ukupan broj bakterija }\end{array}$ & $4.13 \pm 0.16$ & $6.09 \pm 0.52$ & $7 \pm 0$ & $8.04 \pm 0.91$ & $1.26 \pm 0.17$ & $1.33 \pm 0.17$ & $6.83 \pm 0.02$ & $7 \pm 0$ \\
\hline Micrococcaceae & ND & ND & ND & ND & ND & ND & ND & ND \\
\hline E. coli & ND & ND & ND & $3.11 \pm 0.16$ & ND & ND & $1.98 \pm 003$ & ND \\
\hline Enterococcus spp. & $2 \pm 0$ & $5.67 \pm 0.06$ & $3.04 \pm 0.07$ & $3.35 \pm 0.31$ & $2.13 \pm 0.08$ & $4.74 \pm 0.04$ & $5.04 \pm 0.04$ & $3.94 \pm 0.03$ \\
\hline S. aureus & $2.24 \pm 0.21$ & ND & ND & ND & ND & ND & ND & ND \\
\hline Pseudomonas spp. & ND & ND & ND & ND & ND & ND & ND & ND \\
\hline $\begin{array}{l}\text { Sulphite-reducing } \\
\text { bacteria }\end{array}$ & ND & ND & ND & ND & ND & ND & ND & ND \\
\hline $\begin{array}{l}\text { Clostridium } \\
\text { perfringens }\end{array}$ & ND & ND & ND & ND & ND & ND & ND & ND \\
\hline Enterobacteriace & ND & ND & ND & $5.04 \pm 0.4$ & ND & $3.8 \pm 0.29$ & $2.67 \pm 0.31$ & ND \\
\hline Salmonella spp. & ND & ND & ND & ND & ND & ND & ND & ND \\
\hline L. monocytogenes & ND & ND & ND & ND & ND & ND & ND & ND \\
\hline
\end{tabular}

Legend/Legenda: ND - not detected/nije otkriven

Table 2. Microbiological contamination of processing environment, food contact surfaces, equipment and workers' hands in the household $\mathrm{B}$ during the meat production process ( $\mathrm{MS} \pm \mathrm{Sd}, \log _{10} \mathrm{CFU} / \mathrm{cm}^{2}$ ).

Tabela 2. Mikrobiološka kontaminacija radne sredine, radnih površina, opreme i ruku radnika u okviru domaćinstva $\mathrm{B}$ tokom proizvodnje mesa $\left(\mathrm{MS} \pm \mathrm{Sd}, \log _{10} \mathrm{cfu} / \mathrm{cm}^{2}\right)$

\begin{tabular}{|c|c|c|c|c|c|c|c|c|c|c|c|}
\hline $\begin{array}{l}\text { Microbiological } \\
\text { contamination/ } \\
\text { Mikrobiološka } \\
\text { kontaminacija }\end{array}$ & $\begin{array}{c}\text { Workers' } \\
\text { hands/ } \\
\text { Ruke } \\
\text { radnika }\end{array}$ & $\begin{array}{c}\text { Workers' } \\
\text { hands/ } \\
\text { Ruke } \\
\text { radnika }\end{array}$ & $\begin{array}{c}\text { Workers' } \\
\text { hands/ } \\
\text { Ruke } \\
\text { radnika }\end{array}$ & $\begin{array}{l}\text { Saw/ } \\
\text { Testera }\end{array}$ & $\begin{array}{c}\text { Saw after } \\
\text { cutting/ } \\
\text { Testera } \\
\text { posle } \\
\text { klanja }\end{array}$ & $\begin{array}{l}\text { Knife/ } \\
\text { Nožz }\end{array}$ & $\begin{array}{c}\text { Knife } \\
\text { after } \\
\text { cutting/ } \\
\text { Nož posle } \\
\text { klanja }\end{array}$ & \begin{tabular}{|} 
Chopper/ \\
Mašina \\
za sečenje
\end{tabular} & \begin{tabular}{|c|} 
Chopper \\
after \\
Cutting/ \\
Mašina \\
za sečenje \\
posle \\
sečenja
\end{tabular} & $\begin{array}{l}\text { Apron/ } \\
\text { Kecelja }\end{array}$ & $\begin{array}{l}\text { Drain/ } \\
\text { Odvod }\end{array}$ \\
\hline $\begin{array}{l}\text { Total viable count/ } \\
\text { Ukupno bakterija }\end{array}$ & $3.6 \pm 0.53$ & $4.05 \pm 1.69$ & $3.72 \pm 0.09$ & $5.83 \pm 0.56$ & $6.29 \pm 0.03$ & $5.58 \pm 0.17$ & $6.1 \pm 0.35$ & $2.57 \pm 0.24$ & $6.44 \pm 0.17$ & $6.46 \pm 0.19$ & $7.19 \pm 0.15$ \\
\hline Micrococcaceae & ND & ND & ND & ND & ND & ND & ND & ND & ND & ND & ND \\
\hline E. coli & ND & ND & ND & ND & $3.41 \pm 0.36$ & ND & $1.87 \pm 0$ & ND & $3.41 \pm 0.23$ & ND & $3 \pm 0$ \\
\hline Enterococcus spp. & ND & ND & ND & $4.28 \pm 0.25$ & $4.45 \pm 0.08$ & ND & $3.66 \pm 0.16$ & ND & $4.39 \pm 0.05$ & ND & $3.33 \pm 0.28$ \\
\hline S. aureus & ND & ND & ND & ND & ND & ND & ND & ND & ND & ND & ND \\
\hline Pseudomonas spp. & ND & ND & ND & ND & ND & ND & ND & ND & ND & ND & ND \\
\hline $\begin{array}{l}\text { Sulphite-reducing } \\
\text { bacteria }\end{array}$ & ND & ND & ND & ND & ND & ND & ND & ND & ND & ND & ND \\
\hline $\begin{array}{l}\text { Clostridium } \\
\text { perfringens }\end{array}$ & ND & ND & ND & ND & ND & ND & ND & ND & ND & ND & ND \\
\hline Enterobacteriaceae & ND & ND & ND & $3.52 \pm 0.17$ & $5.07 \pm 0.5$ & ND & $5.73 \pm 0.2$ & ND & $4.64 \pm 0.06$ & ND & $2.37 \pm 0$ \\
\hline Salmonella spp. & ND & ND & ND & ND & ND & ND & ND & ND & ND & ND & ND \\
\hline L.monocytogenes. & ND & ND & ND & ND & ND & ND & ND & ND & ND & ND & $2.28 \pm 0.02$ \\
\hline
\end{tabular}

Legend/Legenda: ND - not detected/nije otkriven 
Table 3. Microbiological contamination of processing environment, food contact surfaces, equipment and workers' hands in the household A during preparation of the filling ( $\mathrm{MS} \pm \mathrm{Sd}, \log _{10} \mathrm{CFU} / \mathrm{cm}^{2}$ )

Tabela 3. Mikrobiološka kontaminacija radne sredine, radnih površina, opreme i ruku radnika u okviru domaćinstva A tokom pripreme nadeva ( $\left.\mathrm{MS} \pm \mathrm{Sd}, \log _{10} \mathrm{cfu} / \mathrm{cm}^{2}\right)$

\begin{tabular}{|c|c|c|c|c|c|c|c|c|c|}
\hline $\begin{array}{l}\text { Microbiological } \\
\text { contamination/ } \\
\text { Mikrobiološka } \\
\text { kontaminacija }\end{array}$ & $\begin{array}{c}\text { Mincing } \\
\text { machine } \\
\text { (beginning)/ } \\
\text { Mašina za } \\
\text { mlevenje } \\
\text { (početak) }\end{array}$ & $\begin{array}{c}\text { Mincing } \\
\text { machine } \\
\text { (operation)/ } \\
\text { Mašina za } \\
\text { mlevenje } \\
\text { (rad) }\end{array}$ & $\begin{array}{c}\begin{array}{c}\text { Stuffing } \\
\text { machine } \\
\text { (beginning)/ }\end{array} \\
\text { Mašina za } \\
\text { punjenje } \\
\text { (početak) }\end{array}$ & $\begin{array}{c}\text { Stuffing } \\
\text { machine } \\
\text { (operation)/ } \\
\text { Mašina za } \\
\text { punjenje } \\
\text { (rad) }\end{array}$ & $\begin{array}{l}\text { Casing/ } \\
\text { Crevo }\end{array}$ & $\begin{array}{l}\text { Casing with } \\
\text { the filling/ } \\
\text { Crevo sa } \\
\text { punjenjem }\end{array}$ & $\begin{array}{l}\text { Workers' } \\
\text { hands } \\
\text { during } \\
\text { grinding/ } \\
\text { Ruke } \\
\text { radnika } \\
\text { tokom } \\
\text { mlevenja }\end{array}$ & $\begin{array}{l}\text { Workers' } \\
\text { hands with } \\
\text { spices/Ruke } \\
\text { radnika sa } \\
\text { začinima }\end{array}$ & $\begin{array}{l}\text { Drain/ } \\
\text { Odvod }\end{array}$ \\
\hline $\begin{array}{l}\text { Total viable count/ } \\
\text { Ukupno bakterija }\end{array}$ & $4.83 \pm 0.26$ & $6.64 \pm 0.05$ & $3.23 \pm 0.06$ & $6.77 \pm 0.07$ & $6.75 \pm 0.13$ & $6.75 \pm 0.13$ & $6.69 \pm 0.05$ & $6.94 \pm 0.1$ & $6.84 \pm 0.06$ \\
\hline Micrococcaceae & ND & ND & ND & ND & ND & ND & ND & ND & ND \\
\hline E. coli & ND & ND & ND & $1.97 \pm 0.03$ & ND & ND & ND & $2 \pm 0$ & ND \\
\hline Enterococcus spp. & ND & $5.27 \pm 0.25$ & ND & $4.52 \pm 0.06$ & $4.03 \pm 0.05$ & $4.89 \pm 0.06$ & $4.72 \pm 0.05$ & $5 \pm 0$ & $3.28 \pm 0.12$ \\
\hline Staphylococcus aureus & ND & $2 \pm 0$ & ND & ND & ND & ND & $3.31 \pm 0.21$ & $3 \pm 0$ & ND \\
\hline Pseudomonas spp. & ND & ND & ND & ND & ND & ND & ND & ND & ND \\
\hline Sulphite-reducing bacteria & ND & ND & ND & ND & ND & ND & ND & ND & ND \\
\hline Clostridium perfringens & ND & ND & ND & ND & ND & ND & ND & ND & ND \\
\hline Enterobacteriaceae & ND & ND & ND & $1.32 \pm 0.04$ & ND & ND & ND & ND & $2.33 \pm 0.05$ \\
\hline Salmonella spp. & ND & ND & ND & ND & ND & ND & ND & ND & ND \\
\hline Listeria monocytogenes & ND & ND & ND & 2. $03 \pm 0$ & ND & ND & ND & ND & ND \\
\hline
\end{tabular}

Legend/Legenda: ND - not detected/nije otkriven

Table 4. Microbiological contamination of processing environment, food contact surfaces, equipment and workers' hands in the household B during preparation of the filling ( $\mathrm{MS} \pm \mathrm{Sd}, \log _{10} \mathrm{CFU} / \mathrm{cm}^{2}$ )

Tabela 4. Mikrobiološka kontaminacija radne sredine, radnih površina, opreme i ruku radnika u okviru domaćinstva $\mathrm{B}$ tokom pripreme nadeva $\left(\mathrm{MS} \pm \mathrm{Sd}, \log _{10} \mathrm{cfu} / \mathrm{cm}^{2}\right)$

\begin{tabular}{|c|c|c|c|c|c|c|c|c|c|}
\hline $\begin{array}{l}\text { Microbiological } \\
\text { contamination/ } \\
\text { Mikrobiološka } \\
\text { kontaminacija }\end{array}$ & $\begin{array}{c}\text { Mincing } \\
\text { machine } \\
\text { (beginning)/ } \\
\text { Mašina za } \\
\text { mlevenje } \\
\text { (početak) }\end{array}$ & $\begin{array}{c}\text { Mincing } \\
\text { machine } \\
\text { (operation)/ } \\
\text { Mašina za } \\
\text { mlevenje } \\
\text { (rad) }\end{array}$ & $\begin{array}{c}\text { Stuffing } \\
\text { machine } \\
\text { (beginning)/ } \\
\text { Mašina za } \\
\text { punjenje } \\
\text { (početak) }\end{array}$ & $\begin{array}{c}\text { Stuffing } \\
\text { machine } \\
\text { (operation)/ } \\
\text { Mašina za } \\
\text { punjenje } \\
\text { (rad) }\end{array}$ & $\begin{array}{c}\text { Workers' } \\
\text { hands after } \\
\text { cutting the } \\
\text { meat/ } \\
\text { Ruke } \\
\text { radnika } \\
\text { posle } \\
\text { sečenja } \\
\text { mesa }\end{array}$ & $\begin{array}{l}\text { Workers' } \\
\text { hands after } \\
\text { cutting the } \\
\text { meat/ } \\
\text { Ruke } \\
\text { radnika } \\
\text { posle } \\
\text { sečenja } \\
\text { mesa }\end{array}$ & $\begin{array}{c}\text { Workers' } \\
\text { hands after } \\
\text { cutting the } \\
\text { meat/ } \\
\text { Ruke } \\
\text { radnika } \\
\text { posle } \\
\text { sečenja } \\
\text { mesa }\end{array}$ & $\begin{array}{l}\text { Workers' } \\
\text { hands after } \\
\text { mixing the } \\
\text { filling/ } \\
\text { Ruke } \\
\text { radnika } \\
\text { posle } \\
\text { punjenja }\end{array}$ & $\begin{array}{l}\text { Drain/ } \\
\text { Odvod }\end{array}$ \\
\hline $\begin{array}{l}\text { Total viable count/ } \\
\text { Ukupno bakterija }\end{array}$ & $3.18 \pm 0.14$ & $6.56 \pm 0.08$ & $2.21 \pm 0.02$ & $4.21 \pm 0.26$ & $6.21 \pm 0.62$ & $6.1 \pm 0.19$ & $5.2 \pm 0.17$ & $6.75 \pm 0.12$ & $7.19 \pm 0.15$ \\
\hline Micrococcaceae & ND & ND & ND & ND & ND & ND & ND & ND & ND \\
\hline E. coli & ND & ND & ND & ND & ND & ND & ND & ND & $3 \pm 0$ \\
\hline Enterococcus spp. & ND & $3.33 \pm 0.24$ & ND & $3.57 \pm 0.27$ & ND & $4.3 \pm 0.11$ & $4.14 \pm 0.36$ & $4.3 \pm 0.3$ & $3.33 \pm 0.28$ \\
\hline Staphylococcus aureus & ND & ND & ND & ND & ND & ND & ND & ND & ND \\
\hline Pseudomonas spp. & ND & ND & ND & ND & ND & ND & ND & ND & ND \\
\hline $\begin{array}{l}\text { Sulphite-reducing } \\
\text { bacteria }\end{array}$ & ND & ND & ND & ND & ND & ND & ND & ND & ND \\
\hline Clostridium perfringens & ND & ND & ND & ND & ND & ND & ND & ND & ND \\
\hline Enterobacteriaceae & ND & $2.33 \pm 0.58$ & ND & ND & $2.33 \pm 0$ & $2.33 \pm 0$ & ND & $2.11 \pm 0.58$ & $2.37 \pm 0$ \\
\hline Salmonella spp. & ND & ND & ND & ND & ND & ND & ND & ND & ND \\
\hline Listeria monocytogenes & ND & $2.02 \pm 0.46$ & ND & ND & ND & ND & ND & ND & ND \\
\hline
\end{tabular}

Legend/Legenda: ND - not detected/nije otkriven 
Table 5. Microbiological contamination of sausage batter and final product after a drying process $(\mathrm{X} \pm \mathrm{SD}$, $\left.\log _{10} \mathrm{CFU} / \mathrm{cm}^{2}\right)$

Tabela 5. Mikrobiološka kontaminacija nadeva i kobasica nakon procesa sušenja $\left(\mathrm{X} \pm \mathrm{SD}, \log _{10} \mathrm{CFU} / \mathrm{cm}^{2}\right)$

\begin{tabular}{|l|c|c|c|c|c|c|}
\hline $\begin{array}{l}\text { Sample/ } \\
\text { Uzorak }\end{array}$ & $\begin{array}{l}\text { Total viable count/ } \\
\text { Ukupno bakterija }\end{array}$ & Micrococcaceae & Enterococcus spp. & $\begin{array}{c}\text { Lactic Acid } \\
\text { Bacteria }\end{array}$ & Enterobacteriaceae & L. monocytogenes \\
\hline Batter A/Masa A & $7.03 \pm 0.05$ & $4.23 \pm 0.42$ & $3.24 \pm 0.24$ & $\mathrm{ND}$ & $3.89 \pm 0.02$ & $\mathrm{ND}$ \\
\hline Batter B/Masa B & $7.05 \pm 0.07$ & $4.51 \pm 0.45$ & $3.19 \pm 0.17$ & $\mathrm{ND}$ & $4.19 \pm 0.12$ & $\mathrm{ND}$ \\
\hline $\begin{array}{l}\text { Sausage A1/ } \\
\text { Kobasica A1 }\end{array}$ & $4.19 \pm 0.22$ & $2.75 \pm 0.35$ & $<2$ & $5.3 \pm 0.15$ & $\mathrm{ND}$ & $\mathrm{ND}$ \\
\hline $\begin{array}{l}\text { Sausage A2/ } \\
\text { Kobasica A2 }\end{array}$ & $4.28 \pm 0.23$ & $3.4 \pm 0.22$ & $<2$ & $6.4 \pm 0.22$ & $\mathrm{ND}$ & $\mathrm{ND}$ \\
\hline $\begin{array}{l}\text { Sausage B1/ } \\
\text { Kobasica B1 }\end{array}$ & $4.5 \pm 0.05$ & $2.64 \pm 0.1$ & $<2$ & $5.7 \pm 0.15$ & $\mathrm{ND}$ & $\mathrm{ND}$ \\
\hline $\begin{array}{l}\text { Sausage B2/ } \\
\text { Kobasica B2 }\end{array}$ & $4.31 \pm 0.02$ & $2.62 \pm 019$ & $<2$ & $6.2 \pm 0.30$ & $\mathrm{ND}$ & $\mathrm{N}$ \\
\hline
\end{tabular}

Legend/Legenda: ND - not detected/nije otkriven

B (Table 2) showed similar situation with regard to the presence of microorganisms (aerobic bacteria, E. coli, enterococci and Enterobacteriaceae). The working surfaces, machines, tools and worker's hands had total aerobic counts between (chopper) and $7.19 \pm 0.15 \log _{10} \mathrm{cfu} / \mathrm{cm}^{2}$. For E. coli, contamination level was $1.87 \pm 00 \quad \log _{10} \mathrm{cfu} / \mathrm{cm}^{2}$, $3.41 \pm 0.23 \log _{10} \mathrm{cfu} / \mathrm{cm}^{2}, 3 \pm 0 \quad \log _{10} \mathrm{cfu} / \mathrm{cm}^{2}$, respectively. Enterobacteriaceae were found in six samples, with maximum of $5.73 \pm 0.2 \log _{10} \mathrm{cfu} / \mathrm{cm}^{2}$ (knife after cutting). The presence of L. monocytogenes was detected in swabs from the drain $2.28 \pm 0.2 \log _{10} \mathrm{cfu} / \mathrm{cm}^{2}$ (Table 2). In households A and $\mathrm{B}$, during preparation of the filling (Tables 3 and 4 ), the presence of L. monocytogenes was detected in swabs from the stuffing $\left(2.03 \pm 0 \log _{10} \mathrm{cfu} / \mathrm{cm}^{2}\right)$ and mincing machine $\left(2.02 \pm 0.46 \log _{10} \mathrm{cfu} / \mathrm{cm}^{2}\right)$. Also, L. monocytogenes was detected in a sausage batter A $\left(2.07 \pm 1270.07 \log _{10} \mathrm{cfu} / \mathrm{cm}^{2}\right)$ and sausage batter B $\left(2.08 \pm 0.08 \log _{10} \mathrm{cfu} / \mathrm{cm}^{2}\right)$ (Table 5).

In regard to the final product - sausage after drying process (Table 5), the presence of aerobic bacteria, micrococci, enterococci and Lactic Acid Bacteria (LAB) was detected while other groups of bacteria were not detected.

\section{Discussion}

Many authors support the belief that the microorganisms present in traditional sausages are derived from the raw materials or from the manufacturing (Talon et al., 2007). This microbiota is usually referred to as "house flora". While the microbiota isolated from traditional sausages is well described, the resident microbiota in the environment of the processing unit is still poorly known. The presence of aerobic bacteria, enterobacteriaceae, enterococci and L. monocytogenes in A and B fillings, most probably resulted from the cross contamination of the sausage batter either with working surfaces or after the meat mincing and addition of spices; that is a consequence of the specific filling preparation technique by manual mixing on the wooden table for ca. 15-30 min (Ikonić et al., 2010). Generally, $L$. monocytogenes and $S$. aureus were detected in 6.97 and $9.30 \%$, respectively, while $E$. coli was found in $18.60 \%$. Sausage samples at the end of the production cycle $\left(270^{\text {th }}\right.$ day) were safe in regard to the presence of bacteria populations from the working environment, such as: aerobic bacteria, Micrococcaceae, Lactic acid bacteria and Enterococcus. The results are in accordance with the results obtained by Lebert et al. (2007), Janković et al. (2013), Lakićević et al. (2014). Several critical points were identified such as the drain, saws, workers' hands, mincing and stuffing machines. The current study revealed that the majority of the sampling sites (control point) tested were $\left(2\right.$ to $\left.6 \log \mathrm{cfu} / \mathrm{cm}^{2}\right)$ contaminated by spoilage flora (Enterobacteriaceae) with knives and saws, mincing machines (Listeria monocytogenes), workers' hands (Staph. aureus, E. coli), which surely indicates an inappropriate slaughtering process, and to a low level of personal hygiene. Detection of Listeria monocytogenes can be considered as a useful indicator of a deterioration in hygiene or process conditions during food production. Unclean, insufficiently or inadequately cleaned pieces of equipment 
have often been identified as a source of pathogens. The results are unique and crucial for the improvement of hygiene control systems in traditional meat processing units (Talon et al., 2007).

\section{Conclusion}

Traditional dry sausages rely on natural contamination by environmental flora. This contamination occurs during slaughtering and increases during manufacturing. The results, during the production of the Petrovská klobása in the traditional manner, showed that processing units were colonised at various levels by spoilage and technological microflora with excessive contamination levels. Sporadic contamination by pathogenic microflora was recorded. $L$. monocytogenes and $S$. aureus were detected in 6.97 and $9.30 \%$ of the samples, respectively, while $E$. coli was enumerated in $18.60 \%$ of the samples. The variability of the contamination emphasized the different

\section{References}

Ikonić P., Petrović Lj., Tasić T., Jokanović M., Savatić S., Džinić N., Ikonić B., 2010. Drying kinetics of Petrovská klobása ripened in traditional and industrial conditions. In: Proc. 2nd Workshop Feed-to-Food - XII International Meat Technology Symposium NODA 2010, Meat technology, quality and safety, Novi Sad, 107-116.

Janković V., Petrović Lj., Lakićević B., Matekalo Sverak V., Lilić S., Mitrović R., 2013. Determination of typical house flora during production process of the Petrovská klobása. African Journal of Microbiology Research, 7, 32, 4130-4137.

Lakićević B., Velebit B., Janković V., Spirić D., Baltić T., Mitrović R., Babić J., 2014. Taq Man Real Time PCR detection of Listeria monocytogenes: a study of enrichment incubation time affecting sensitivity in experimental dry fermented sausages. Tehnologija mesa, 55, 1, 2014.

Lebert I., Leroy S., Giammarinaro P., Lebert A., Chacornac JP., Bover-Cid S., Vidal-Carou M. C., Talon R., 2007. Diversity of microorganisms in the environment and dry fermented sausages of small traditional french processing units. Meat Science, 76, 1,112-122. cleaning, disinfecting and manufacturing practices routinely followed by these (A and B households) small-scale processing units. The technological flora (coagulase negative staphylococci and lactic acid bacteria) were both in the environment and in products. Enterococci were present all along the manufacturing period. Examination of the hygienic status of the processing environment, equipment, raw materials and food safety criteria of the final product provides an overview of growth trends and the disappearance of bacterial populations.

Further studies will be carried out to detail phenotypic, genotypic and physiological characterization of the isolated strains of staphylococci (CNS) and LAB from the Petrovská klobása with additional purpose of creating Serbian bank of autochthonous functional starter cultures specific for industrial production of the Petrovská klobása. In this way, the product will meet all regulations, as needed on a broad market, and it will remain autochthonous, safe and recognizable.

Leroy S., Giammarinaro P., Chacornac J. P., Lebert I., Talon R., 2010. Biodiversity of indigenous staphylococci of naturally fermented dry sausages and manufacturing environments of small-scale processing units. Food Microbiology, 27, 2, 294-301.

Šojić B., Petrović Lj., Tomović V., Džinić N., Mandić A., Škaljac S., Jokanović R., Ikonić P., Tasić T., Sedej I., 2014. Effect of Ripening Conditions and Storage Time on Oxidative and Sensory Stability of Petrovská Klobása. International Journal of Biological, Veterinary, Agricultural and Food Engineering, 8, 5.

Talon R., Lebert I., Lebert A., Leroy S., Garriga M., Aymerich T., Drosinos EH., Zanardi E., Ianieri A., Fraqueza M. J., Patarata L., Laukova A., 2007. Traditional dry fermented sausages produced in small -scale processing units in Mediterranean countries and Slovakia. Meat Science, 77, 4, 570-579.

Tasić T., 2012. Producing of Biogenic amines in traditional dry fermented sausage Petrovská klobása, during safety and quality standardization process. PhD thesis, Faculty of Technology, University of Novi Sad, Serbia. 


\title{
Mikrobiološki ekosistem malih proizvodnih jedinica tokom proizvodnog ciklusa petrovačke kobasice
}

\author{
Janković Vesna, Petrović Ljiljana, Džinić Natalija, Lakićević Brankica, Šarić Ljubiša, Škaljac Snežana, \\ Šojić Branislav, Jokanović Marija
}

R e z i m e: Proizvodnja tradicionalnih suvih-fermentisanih kobasica je u direktnoj vezi sa mikrobiološkim ekosistemom radne sredine (house flora), čije definisanje ima značajnu ulogu u mikrobiološkoj stabilnosti i bezbednosti gotovog proizvoda. U okviru rada, prezentovani su rezultati koji daju uvid u diverzitet microbiota u malim proizvodnim jedinicama, tokom proizvodnog ciklusa petrovačke kobasice. Test uzorci su sakupljeni u dva seoska domaćinstva u Bačkom Petrovcu, gde je izvršena priprema petrovačke kobasice na tradicionalan način. Ispitivanjem su obuhvaćena ukupno 43 uzorka. Generalno, Listeria monocytogenes i Staphylococcus aureus su detektovani u 6,97 i 9,30\%, respektivno, dok je Escherichia coli detektovana u 18,60\% uzoraka. Uzorci kobasica su na kraju perioda skladištenja (270. dan) bili bezbedni uz prisustvo sledećih grupa bakterija: aerobne bakterije, Micrococcaceae, bakterije mlečne kiseline i Enterococcus spp.

Ključne reči: Petrovačka kobasica, trend rasta, radna sredina.

Paper received: 10.10 .2014 .

Paper corrected: 20.10.2014.

Paper accepted: 21.10.2014. 